\title{
Images in Clinical Tropical Medicine Concurrent COVID-19 and Tuberculosis in an Immigrant Worker Presenting with Hemoptysis
}

\author{
Toshinori Sahara* and Kazuhisa Yokota \\ Department of Infectious Diseases, Ebara Hospital, Tokyo Metropolitan Health and Hospital Corporation, Tokyo, Japan
}

A 59-year-old man was referred to our hospital by a regional public health center for the treatment of coronavirus disease 2019 (COVID-19), which was diagnosed by the nasopharyngeal swab polymerase chain reaction test. The patient, who is immigrant worker from Nepal at an Indian restaurant in Japan, had acute-onset fever, productive cough with hemoptysis, and taste and smell disorders for 8 days before admission. He had no past medical history including tuberculosis (TB), and no subacute or chronic symptoms to suspect TB. None of his family members had TB. When he was admitted, he had a body temperature of $37.4^{\circ} \mathrm{C}$, a respiratory rate of 14 breaths/min, an oxygen saturation level of $100 \%$, and his chest radiograph showed clear lungs (Figure 1). He used acetaminophen and dextromethorphan hydrobromide hydrate to control his fever and cough, but his body temperature proceeded to exceed $38^{\circ} \mathrm{C}$ for 5 days after hospitalization, his productive cough with hemoptysis worsened, and although his oxygen saturation level did not decline, he gradually lost his appetite. We therefore elected to examine his sputum at day 5 after admission. Initial acid-fast stain smears were negative for 3 consecutive days, but the patient finally tested positive for TB by our in-house laboratory polymerase chain reaction analysis of his sputum. Chest computed tomographic scans performed on day 8 after admission revealed bilateral ground-glass opacities, which are typical for COVID-19 patients, and a tree-in-bud pattern in the left upper lobe showing active TB (Figure 2). There were no computed tomographic findings to suspect hilar and mediastinal lymphadenopathy.

After diagnosis of TB, the patient was treated using four standard drugs (rifampicin, isoniazid, ethambutol, and
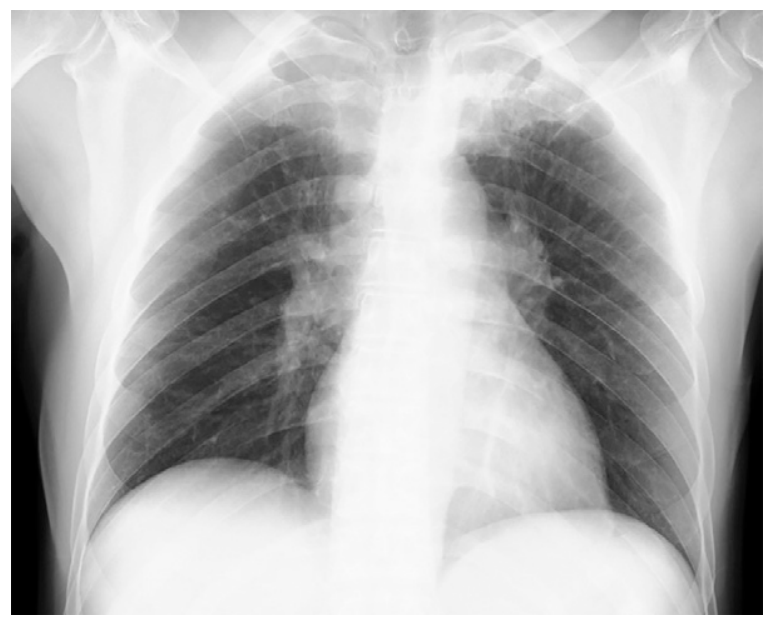

FIGURE 1. Chest X-ray on admission.

\footnotetext{
* Address correspondence to Toshinori Sahara, 4-5-10 HigashiYukigaya, Otaa-ku, Tokyo 145-0065 Japan. E-mail: saharakotetu@gmail.com
}
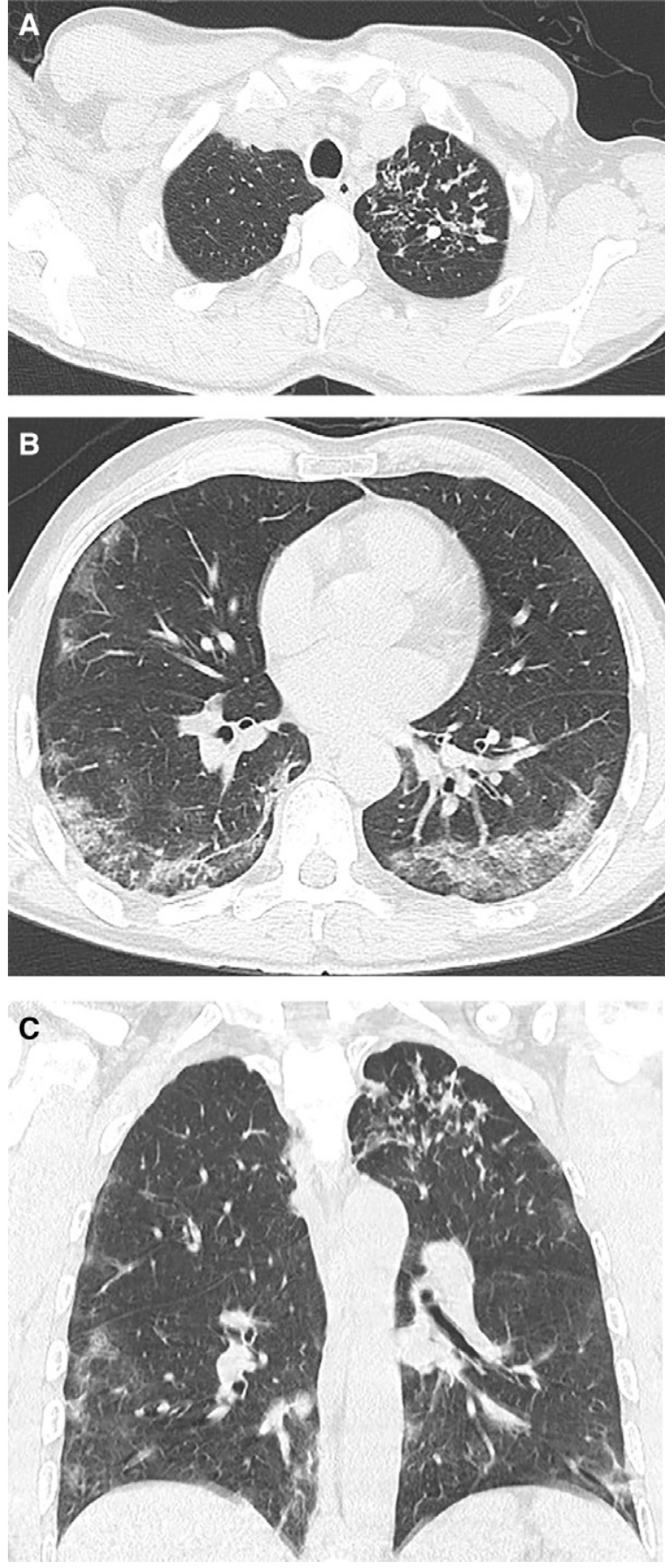

FIGURE 2. (A) Tree-in-bud signs in the left upper lobe. (B) Bilateral ground-glass opacities in a peripheral distribution. (C) Coronal computed tomographic scan shows both tree-in-bud pattern and peripheral ground-glass opacities. 
pyrazinamide) from day 9 after admission. His fever decreased the following day, his productive cough improved gradually, and his appetite returned. The sputum culture subsequently tested positive for Mycobacterium tuberculosis, which was sensitive to every anti-TB drug.

The prevalence of hemoptysis in patients with COVID-19 and TB has been reported as $2 \%^{1}$ and as approximately $8 \% .^{2,3}$ Elise et al. ${ }^{4}$ reported a COVID-19 case with acuteonset hemoptysis, and another report indicated that the duration of hemoptysis in patients with COVID-19 may be less than 10 days. $^{5}$

This case exemplifies the importance of being aware of possible concurrent infections in patients with COVID-19, and that correct assessment of patients at appropriate times is essential to identify co-infections. The epidemiological background of TB, clinical pattern of symptom onset, and period of hemoptysis are key to recognizing concurrent infection of TB among patients with COVID-19 presenting hemoptysis.

Received April 29, 2021. Accepted for publication June 10, 2021.

Published online July 16, 2021.
Acknowledgment: We thank all of the staff in the COVID-19 ward at Ebara Hospital for their assistance with clinical management.

Authors' addresses: Toshinori Sahara and Kazuhisa Yokota, Department of Infectious Diseases, Ebara Hospital, Tokyo Metropolitan Health and Hospital Corporation, Tokyo, Japan, E-mails: saharakotetu@gmail.com and yokota77@gmail.com.

This is an open-access article distributed under the terms of the Creative Commons Attribution (CC-BY) License, which permits unrestricted use, distribution, and reproduction in any medium, provided the original author and source are credited.

\section{REFERENCES}

1. Fu L et al., 2020. Clinical characteristics of coronavirus disease 2019 (COVID-19) in China: a systematic review and meta-analysis. J Inf Secur 80: 656-665.

2. Middleton JR, Sen P, Lange M, 1997. Death-producing hemoptysis in tuberculosis. Chest 72: 601-604.

3. Leung AN, 1999. Pulmonary tuberculosis: the essentials. Radiology 210: 307-322.

4. Elise $\mathrm{P}$ et al., 2020. Hemoptysis as the first presentation of COVID19: a case report. BMC Pulm Med 20: 275.

5. Shi F, Yu Q, Huang W, Tan C, 2020. 2019 novel coronavirus (COVID-19) pneumonia with hemoptysis as the initial symptom: CT and clinical features. Korean J Radiol 21: 537-540. 